\title{
CXCR4 receptor blockage reduces the contribution of tumor and stromal cells to the metastatic growth in the liver
}

\author{
AITOR BENEDICTO, IRENE ROMAYOR and BEATRIZ ARTETA \\ Department of Cellular Biology and Histology, University of The Basque Country, \\ School of Medicine and Nursing, UPV/EHU, Leioa, E-48940 Biscay, Spain
}

Received September 20, 2017; Accepted January 24, 2018

DOI: $10.3892 /$ or.2018.6254

\begin{abstract}
The liver is a common site for the metastatic spread of primary malignancies including colorectal cancer, and liver metastasis is a main cause of death in cancer patients. This is due to the complexity of the interactions taking place in the liver between tumor and stromal cells. In fact, cancer-associated fibroblasts (CAFs) have been shown to support tumor growth through the CXCL12/CXCR4 axis. However, along with cancer cells, myeloid-derived suppressor cells (MDSCs), immature dendritic cells with immunosuppressive potential, also express CXCR4. It has recently been demonstrated that reducing CXCL12 availability in the tumor microenvironment decreases liver metastasis. Therefore, blocking CXCL12 chemokine receptor CXCR4 may be a successful approach to diminish the metastatic spread of colorectal cancer to the liver. However, the subjacent mechanisms by which this chemokine influences the tumor are not fully understood. Thus, in order to uncover the role of CXCR4 during tumor cell/liver fibroblast crosstalk driving liver metastasis, the CXCR4 antagonist AMD3100 was used for in vitro studies and in an in vivo approach using an orthotopic model of liver metastasis in immune competent mice through intrasplenic injection of grafted C26 cells. In vitro blockage of CXCR4 led to an impaired migratory potential of tumor and hepatic stellate cells (HSCs) and a reduced tumor response to CXCL12. In vivo administration of AMD3100 to tumor-bearing mice resulted in attenuated metastatic development in the liver, which was
\end{abstract}

Correspondence to: Dr Beatriz Arteta, Department of Cellular Biology and Histology, University of The Basque Country, School of Medicine and Nursing, Barrio Sarriena s/n, Leioa, E-48940 Biscay, Spain

E-mail: beatriz.arteta@ehu.es

Abbreviations: MDSCs, myeloid-derived suppressor cells; HSCs, hepatic stellate cells; $\alpha$ SMA, alpha smooth muscle actin; CAFs, cancer-associated fibroblasts; LSECs, liver sinusoidal endothelial cells; ECM, extracellular matrix

Key words: liver metastasis, hepatic stellate cells, myeloid-derived suppressor cells, immune suppression, CXCR4, CXCL12 accompanied by an impaired infiltration of $\alpha$ SMA-expressing cells within the tumors. In addition, a reduced $\mathrm{CD} 11^{+} \mathrm{Ly} 6 \mathrm{G}^{+}$ cell count in the liver was directly correlated with a reduction in MDSC numbers in the blood of AMD3100-treated mice compared to the vehicle-treated mice. Therefore, disruption of the CXCR4/CXCL12 axis by CXCR4 antagonist AMD3100 blocked the contribution of both cancer and stromal cells to the metastatic cascade in the liver.

\section{Introduction}

Colorectal cancer remains one of the most prevalent malignancies. While primary tumors are often resectable leading to a better prognosis, the presence of metastatic lesions represent the worst scenario medicine has to cope with. Colorectal cancer metastasizes mainly to the liver and almost $70 \%$ of patients with this cancer present distant invasion at the time of diagnosis. The mechanisms leading to metastatic formation and growth remain poorly characterized, especially those linked with stromal cells, known to facilitate tumor cell escape from the immune system and support tumor growth $(1,2)$. Therefore, a better understanding of the tumor microenvironment is required for a wider comprehension of metastatic disease. One of the receptors expressed in stromal cells, along with tumor cells, is CXCR4, one of the two known receptors for CXCL12, also known as stromal cell-derived factor 1 (SDF-1) (3).

CXCR4 has been described to be upregulated in human melanoma metastasis or colorectal cancer lymph node and liver metastases and has been linked with poor survival (4-6). This receptor has been shown to mediate different pro-metastatic events in tumor cells in vitro, such as invasion and migration and cancer cell extravasation in vivo (7-9). Interestingly, this receptor is not only expressed by tumor cells but also has been found in hepatic stellate cells (HSCs), liver resident cells. Under inflammatory conditions, these cells become activated and trans differentiate into myofibroblast-like cells, supporting fibrosis, liver cancer and liver metastasis (10-12). In the liver, HSCs are the main population of cancer-associated fibroblasts (CAFs), which are the main component of the tumor microenvironment (13) and arise as a modulator of the tumor response. Intriguingly, tumor/fibroblast interaction has been postulated as a relevant event during the progression of cancer with the CXCR4/CXCL12 chemokine axis one of the main catalysts of malignancy (14). Furthermore, HSCs, along with 
liver sinusoidal endothelial cells (LSECs), are one of the main sources of CXCL12 in the liver where they mediate not only the recruitment of CXCR4-expressing tumor cells, but also CXCR4-expressing immune cells.

During cancer development and metastasis formation, the cytotoxicity against cancer cells is compromised due to immune suppression. This pathological process is in part orchestrated by myeloid-derived suppressor cells (MDSCs), CXCR4-expressing immature myeloid cells able to suppress the cytotoxic capacity of T lymphocytes (15). During liver metastasis, these cells are recruited into the organ (16) from the circulation leading to uncontrolled tumor growth and disease progression. In fact, this chemokine and its CXCR4 receptor seem to be relevant for tumor progression, as reported through the reduction of CXCL12 availability and CXCR4 blockage within the tumor microenvironment, reducing liver metastasis in nude mice (17). Moreover, CXCR4 blockage has been confirmed to be effective for reducing in vivo lung cancer metastasis (18), pointing out the interaction of CXCR4 with its lig and CXCL12 as a mediator in this process. However, the cellular and molecular mechanisms by which CXCR4 interaction with CXCL12 drives disease progression remain poorly understood, especially when it comes to stromal cells.

Therefore, the present study aimed to investigate the efficacy of treatment with the CXCR4 antagonist AMD3100 in an orthotopic model of colorectal cancer liver metastasis and the cellular mechanisms involved in the processes disrupted by this antagonist.

\section{Materials and methods}

Animals. Six-week-old male Balb/c mice were obtained from Janvier Labs (France). Housing, care, and experimental conditions were carried out in conformity with institutional guidelines and national and international laws for experimental animal care. The animals were fed a standard chow and had access to water ad libitum. All the proceedings were approved by the Basque Country University Ethics Committee (CEID) in accordance with institutional, national and international guidelines regarding the protection and care of animals used for scientific purposes.

Cell lines. All in vitro and in vivo experiments were conducted using the murine $\mathrm{C} 26$ colon adenocarcinoma (C26) cell line (also known as MCA-26, CT-26) syngeneic with Balb/c mice and purchased from ATCC (LGC Standards S.L.U. Barcelona, Spain). Cells were cultured in RPMI-1640 medium supplemented with $10 \%$ heat-inactivated fetal bovine serum (FBS), penicillin $(10,000 \mathrm{U} / \mathrm{ml})$, streptomycin $(10,000 \mu \mathrm{g} / \mathrm{ml})$ and amphotericin B $(25 \mu \mathrm{g} / \mathrm{ml})$ all purchased from Thermo Fisher Scientific (Waltham, MA, USA).

Primary murine hepatic stellate cell isolation and culture. HSCs were isolated as previously described with some modifications $(19,20)$. Briefly, the liver was perfused with collagenase from Clostridium histolyticum (Sigma-Aldrich, St. Louis, MO, USA) and the resulting cell suspension was subjected to isopycnic centrifugation through a Percoll gradient (GE Healthcare, Chicago, IL, USA). The fraction enriched in HSCs was cultured in culture medium without serum supplemented with antibiotics and antimycotics incubated at $37^{\circ} \mathrm{C}$ in $5 \% \mathrm{CO}_{2}$ overnight before the experimental procedures.

Preparation of conditioned culture medium. Tumor cells were seeded at a concentration of $5 \times 10^{4}$ cells $/ \mathrm{cm}^{2}$ and cultured overnight in RPMI-1640 supplemented with 10\% FBS and antibiotics. Then, the culture medium was substituted with fresh medium supplemented with $1 \%$ FCS. After $24 \mathrm{~h}$, the cell culture supernatant was collected and used as C26-conditioned medium $(\mathrm{C} 26-\mathrm{cm})$ for further studies. HSC conditioned medium was also prepared. To do so, HSCs were cultured at a concentration of $7,5 \times 10^{4}$ cells $/ \mathrm{cm}^{2}$ as previously described (10). Then, culture medium was replaced with fresh medium supplemented with $1 \%$ FBS and HSCs were cultured for an additional $24 \mathrm{~h}$. The supernatant was collected and used as HSC-conditioned medium for further studies. In some cases, HSCs were exposed to C26-cm diluted 2-fold with fresh medium for $24 \mathrm{~h}$. Then, the supernatant was collected and used as tumor-activated HSC-cm (taHSC-cm).

Western blot analyses. CXCR4 expression was analyzed by western blotting in C26 cells and HSCs under several conditions. To do so, the tumor cells were either untreated or treated with HSC-cm or taHSC-cm, and HSCs were either untreated or treated with $\mathrm{C} 26-\mathrm{cm}$. After $48 \mathrm{~h}$ of treatment, the cultured medium was removed and cell lysates were obtained. To obtain cell lysates for protein extraction, the cells were washed twice with cold $1 \mathrm{X}$ phosphate-buffered saline (PBS) before adding $100 \mu \mathrm{l} / 10^{6}$ cells of $1 \mathrm{X}$ Laemmli sample buffer (Bio-Rad, USA) with $1 \% \beta$-mercaptoethanol and $1 \mathrm{M} \mathrm{NaCl}$. Sample electrophoresis was performed using $10 \%$ SDS-PAGE gels and run for 1.5 hat $120 \mathrm{mV}$ on ice. Proteins were transferred onto a nitrocellulose membrane in wet conditions at $380 \mathrm{~mA}$ for 3.5 hon ice. Then, the membranes were incubated with rabbit anti-mouse CXCR4 (1:1,000; cat. no. PA3-305; Thermo Fisher Scientific) overnight at $4^{\circ} \mathrm{C}$ in $5 \%$ milk containing TBS-T (2\% $1 \mathrm{M}$ Tris-HCL pH, $7.510 \% 5 \mathrm{M} \mathrm{NaCl}$, $0.1 \%$ Tween-20), followed by a $1-\mathrm{h}$ incubation with specific goat anti-rabbit biotinylated secondary antibodies (1:2,000; cat. no. 65-6140). Streptavidine-HRP conjugated (1:500) was used for detection. Antibodies and detection systems were purchased from Thermo Fisher Scientific. Finally, the bands were visualized using Luminata ${ }^{\mathrm{TM}}$ Crescendo Western HRP Substrate (Millipore, Billerica, MA, USA). SnapGene software (Syngene, Frederick, MD, USA) was used to measure the protein expression (Each experiment was performed three times).

Flow cytometric analyses. Flow cytometric analyses were carried out for the quantification of CXCR4 expression in C26 cells and quantification of MDSCs in the circulation of tumor-bearing mice. To do so, C26 cells and PMNCs (for collection of PMNCs see later on) were fixed in $70 \%$ ethanol for $10 \mathrm{~min}$ before the unspecific background was blocked by a 30-min incubation in PBS containing 5\% FBS. The cells were incubated for $2 \mathrm{~h}$ with either rat anti-mouse CXCR4 monoclonal antibody (1:500; cat. no. MAB21651; R\&D Systems Inc., Minneapolis, MN, USA), FITC-conjugated rat anti-mouse CD11b (1:500; cat. no. ab24874; Abcam, Cambridge, UK) and rat anti-mouse-Ly6G primary antibody (1:500; cat. no. NBP1-28168; Novus Biologicals, Littleton, CO, USA). 
Then, cells were incubated with Alexa-488 (excitation 488, emission 525) and Alexa-594 (excitation 594, emission 617) conjugated-secondary antibody for CXCR4 and MDSC detection, respectively. Immunolabelled cells were analyzed by flow cytometry (Gallios; Beckman Coulter, Brea, CA, USA). Flow cytometric analyses were performed three times for tumor cells and a pool of 5 mice were used for MDSC quantification.

Cell viability assay. C26 viability was analyzed using PrestoBlue $^{\mathrm{TM}}$ Cell Viability reagent (Life Technologies Inc.). C26 tumor cells were seeded at a concentration of $5 \times 10^{3}$ cells $/ \mathrm{cm}^{2}$ and let expand for $3 \mathrm{~h}$. Afterwards, the tumor cells were incubated with increasing concentrations $(0.1,1$ and $10 \mathrm{nM}$ ) of CXCL12 (OriGene, Rockville, MD, USA) with or without $10 \mu \mathrm{g} / \mathrm{ml}$ AMD3100 (from $2 \mathrm{~h}$ prior to the addition of CXCL12), for $48 \mathrm{~h}$ (AMD3100 was previously diluted in PBS for stock solution). Then, cell viability was quantified by adding PrestoBlue ${ }^{\mathrm{TM}}$ reagent for $120 \mathrm{~min}$. The reaction was measured with the Ascent Multiskan (Labsystems). Cell viability was represented as percentage of the increase respect to cell viability at time 0 (Cell viability assay was carried out three times).

Gelatin zymography. MMP-2 and MMP-9 activity was measured by gelatin zymography in C26 cells or HSC supernatants. The cells were treated with either $1 \mathrm{nM}$ CXCL12, or AMD3100 $(10 \mu \mathrm{g} / \mathrm{ml}$ from $2 \mathrm{~h}$ prior to the addition of CXCL12) or the combination of both for $24 \mathrm{~h}$. Cell culture supernatants were collected and run on $1 \%$ gelatin containing electrophoresis gel. After 2 washes in $2.5 \%$ TritonX-100, the gels were incubated overnight in a developing buffer containing divalent cations for the proper activity of MMPs. After wards, the gel was stained with Coomassie Blue to visualize degradation bands. Images were captured through Quantity One program (Bio-Rad Laboratories, Hercules, CA, USA) (MMP-2 and MMP-9 secretion was measured three times).

Migration assay. For the quantification of the migratory potential of C26 cells and HSCs, modified Boyden chambers were utilized. Briefly, cells were seeded onto type I collagen-coated $8-\mu \mathrm{m}$ diameter pore membrane inserts (Greiner Bio-One GmbH, Frickenhausen, Germany). Cells were incubated in RPMI-1640 supplemented with 1\% FBS and antibiotics and allowed to adhere and expand for $3 \mathrm{~h}$ before the addition of AMD3100 $(10 \mu \mathrm{g} / \mathrm{ml})$ in both upper and lower chambers. After incubation of the cells with AMD3100 for $2 \mathrm{~h}$, the medium was replaced and the cells were treated with either AMD3100 $(10 \mu \mathrm{g} / \mathrm{ml}), 1 \mathrm{nM}$ CXCL12 or a combination of both for $18 \mathrm{~h}$ supplemented with $1 \%$ FBS. Then, the non-migrated cells were gently removed from the upper side of the insert and the remaining cells on the external side of the insert were subjected to $70 \%$ ethanol fixation and DAPI mounting medium for visualization of the nuclei. The number of migrated tumor cells and HSCs was quantified in 10 fields at x10 magnification under Axioscope fluorescence microscope (Carl Zeiss, Oberkochen, Germany). The migratory potential is represented as the percentage of migrated treated cells in respect to the untreated cells (migration studies were performed three times).
Experimental development of liver metastasis. C26 tumor cells were in trasplenically injected at a concentration of $2 \times 10^{5}$ cells in $100 \mu 1$ PBS. Briefly, a thin cut was made in the left flank of each anesthetized mouse and the spleen was exposed. Then, the tumor cell suspension of the grafted C26 colorectal cancer cells was slowly injected in the spleen distal pole. Afterwards, the spleen was relocated and the wound was closed. Mice were treated daily with $5 \mathrm{mg} / \mathrm{kg}$ AMD3100 or vehicle solution starting $24 \mathrm{~h}$ after tumor cell injection. All animals were sacrificed 14 days after tumor cell injection and livers were frozen and kept at $-80^{\circ} \mathrm{C}$ for immunehistochemical analyses or paraffin embedded for hematoxylin and eosin staining and quantification of liver tissue area occupied by the tumor. In vivo metastasis assay was performed in duplicate with 5 animals in each group/experiment ( $\mathrm{n}=10$ each group).

Immunohistochemical analysis. Frozen liver sections $(10-\mu \mathrm{m}$ thick) were analyzed for the quantification of different immune cell populations. Liver sections were stained with specific primary antibodies: Ratanti-mouse CD11b as neutrophil marker (Abcam), ratanti-mouseLy6G as granulocytic marker (Novus Biologicals) and Cy3-conjugated mouse anti-human $\alpha$ SMA (1:500; cat. no. C6198; Sigma-Aldrich) as HSC activation marker. After blocking and incubation with primary antibodies, surface molecules were detected by the use of secondary antibodies conjugated either with Alexa-488 or Alexa-594. For $\alpha$ SMA staining, samples were incubated with rabbit anti-mouse IgG F (ab') (cat. no. 31192; Thermo Fisher Scientific) diluted in PSB for the blockage of endogenous mouse IgGs for $1 \mathrm{~h}$ prior to the primary antibody incubation $(0.15 \mathrm{mg} / \mathrm{ml})$. The number of $\mathrm{CD} 11 \mathrm{~b}^{+} \mathrm{Ly} 6 \mathrm{G}^{+}$cells along with in tratumoral $\alpha$ SMA expression was analyzed in the metastatic livers and quantified by image analyses with ImageJ software (NIH, Bethesda, MD, USA).

Isolation of blood PMNCs. For isolation of PMNCs, mice were anesthetized prior to their sacrifice and the abdominal cavity was opened exposing the cava vein. Sterile PBS-EDTA was inoculated into the cava vein right before extraction of blood using the same syringe. Erythrocytes were discarded using Lympholyte $\mathrm{M}$ (Cederlane, Canada) density gradient and PMNCs and lymphocyte fraction collected for flow cytometry analyses as described above. Results represent the pool of 5 mice/group.

Statistical analyses. Data are expressed as mean \pm standard deviation (SD) of three independent experiments. Statistical analysis was performed using SPSS version 13.0 (SPSS, Inc. Chicago, IL, USA). Individual comparisons were performed using two-tailed, unpaired Student's t-test. Differences were considered to be significant for $\mathrm{P}<0.05$.

\section{Results}

Tumor/stroma crosstalk stimulates CXCR4 expression in C26 cells and HSCs. Tumor/stroma interaction is known to modulate a wide variety of proteins in both cancer and stromal cells (10). As mentioned above, CXCR4 expression is localized in HSCs in the liver and tumor cells. We treated C26 cells with soluble factors derived from either quiescent HSCs or taHSCs, 
A

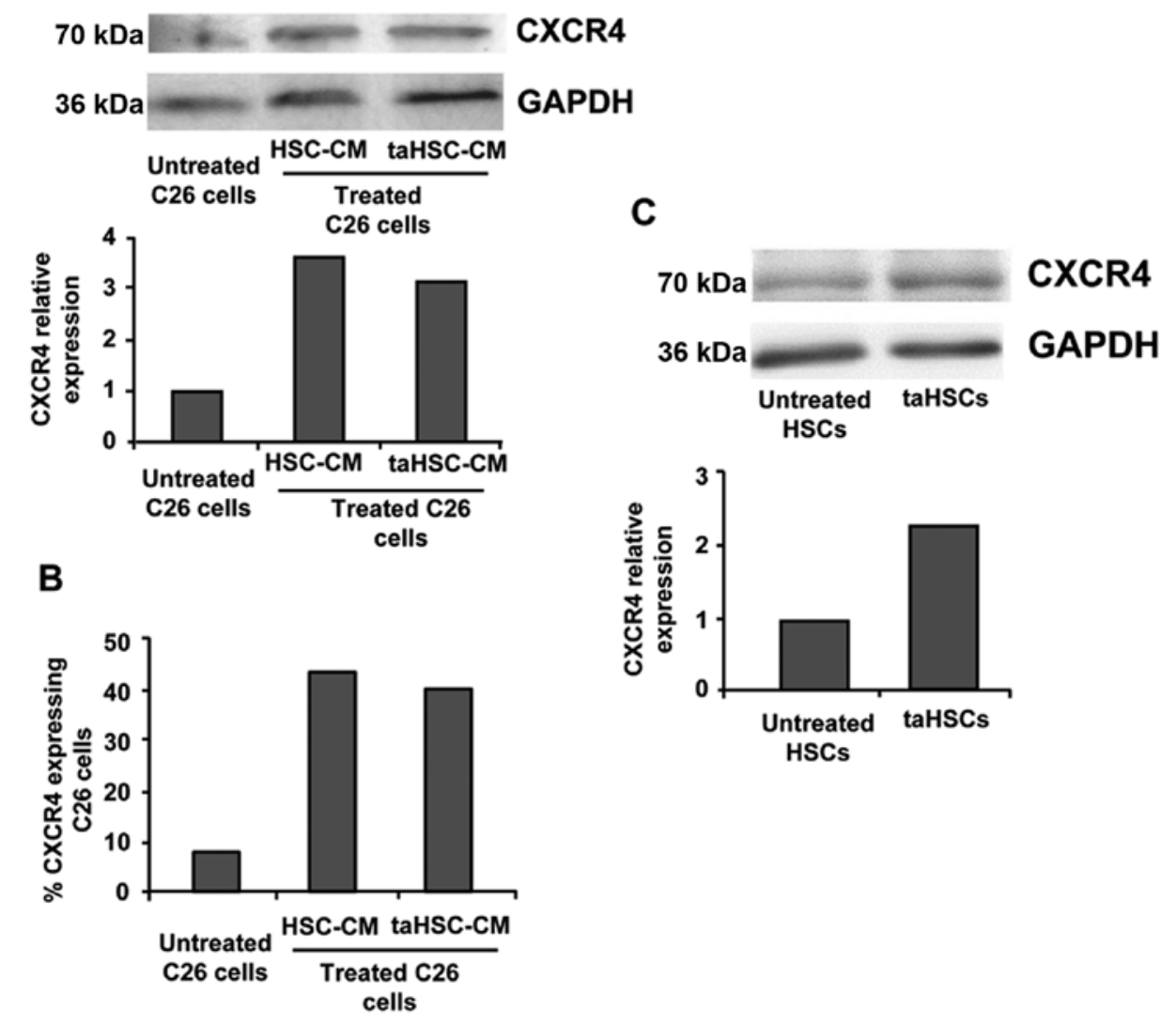

Figure 1. Expression of CXCR4 in C26 cells and HSCs. CXCR4 protein expression was assessed by western blot analysis in tumor cells treated with either HSC-conditioned medium (cm) or tumor-activated HSC (taHSC)-cm supplemented with 1\% FBS for $48 \mathrm{~h}$ (A) and on primary HSCs untreated or taHSCs (C). CXCR4 expression was further analyzed by flow cytometryin C26 cells (B).

which resulted in a 4-fold increase in the CXCR4 expression in $\mathrm{C} 26$ tumor cells compared to untreated $\mathrm{C} 26$ cells when analyzed by western blotting (Fig. 1A) and FACS (Fig. 1B). In the same way, HSCs treated with soluble factors derived from C26 cells showed a 2-fold increased expression of CXCR4 when compared to that of control HSCs (Fig. 1C). Thus, CXCR4 expression was increased in the HSCs and tumor cells after their paracrine interaction.

CXCL12 stimulates the proliferation of C26 cancer cells through CXCR4. Since CXCL12 is known to induce proliferation in CXCR4-expressing cells the effect of CXCL12 on the viability of $\mathrm{C} 26$ cells after treatment with increasing concentrations of CXCL12 was quantified. As shown in Fig. 2A, 0.1 and $1 \mathrm{nM} \mathrm{CXCL12}$ significantly enhanced $\mathrm{C} 26$ viability after a 24-hincubation, while $10 \mathrm{nM}$ did not alter tumor cell proliferation. Blocking of CXCR4 by addition of AMD3100 $(10 \mu \mathrm{g} / \mathrm{ml})$ before CXCL12 addition was able to revert the effect of the chemokine in regards to $\mathrm{C} 26$ cell viability (Fig. 2A). Therefore, CXCR4 mediated the proliferation of CXCL12-stimulated C26 cells.

Effect of CXCL12 on MMP-2 and MMP-9 expression in C26 tumor cells and HSCs. The remodeling of the ECM represents a key event during organ colonization and tumor growth. Tumor and stromal cells contribute to this process by secreting metalloproteases (MMPs), mainly gelatinocytic
MMP-2 and MMP-9. Thus, the presence of these MMPs in the conditioned medium derived from $\mathrm{C} 26$ cells and HSCs was analyzed after the treatment with either CXCL12 $(1 \mathrm{nM})$, AMD3100 $(10 \mu \mathrm{g} / \mathrm{ml})$ or a combination of both. While CXCL12 was unable to increase the levels of any MMP over basal levels, treatment with AMD3100 slightly decreased the MMP-2 and MMP-9 secretion in tumor and stromal cells compared to those present in the supernatants of the control and CXCL12-treated cell cultures (Fig. 2B and C).

CXCR4 mediates the migration of C26 and HSCs towards CXCL12. CXCL12 has been pointed out as a potent chemokine for CXCR4-expressing cells promoting their migration. Thus, we investigated the role of the CXCR4 receptor in the migration of C26 cells and primary HSCs after activation with CXCL12. As shown in Fig. 3A, CXCL12 induced a 4-fold increase in the migratory potential of $\mathrm{C} 26$ cells compared to that of the untreated cells. Blockage of CXCR4 by AMD3100 treatment inhibited the effect of CXCL12 in decreasing the percentage of migrated C26 cells (Fig. 3A). Similarly, CXCL12 activation of primary HSCs also enhanced their migratory potential by 2-fold (Fig. 3B). The effect of this chemokine was partially compromised by using AMD3100, which points to CXCR4 as one of the mediators of CXCL12-induced HSC recruitment into metastatic lesions (Fig. 3B). These results uncover the role of CXCL12/CXCR4 crosstalk during tumor cell and HSC migration. 

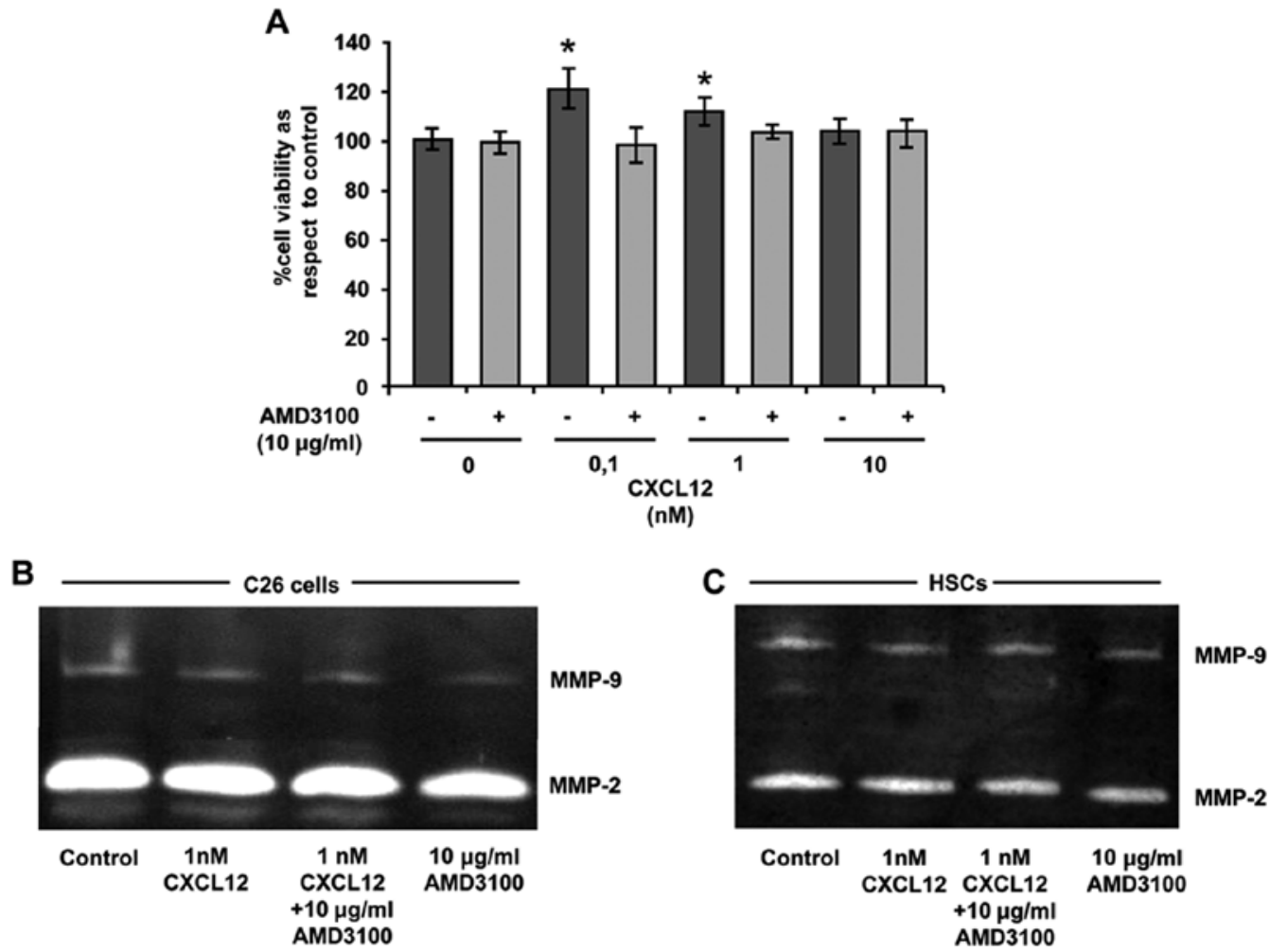

Figure 2. Tumor cell proliferation and MMP secretion by tumor and HSC cells. (A) Effect of CXCL12 ontumor cell viability. (B) Effect of CXCL12 on MMP-2 and MMP-9 secretion by C26 cells. (C) Effect of CXCL12 on MMP-2 and MMP-9 secretion by HSCs. Differences between control and treated cells were considered statistically significant, ${ }^{*} \mathrm{P}<0.05$.

CXCR4 blockage via AMD3100 administration reduces the number and size of metastatic foci in the liver. To further investigate the significance of CXCR4 during metastatic spread, in vivo liver metastasis assay was carried out using colorectal cancer $\mathrm{C} 26$ cell line. Mice were administered with a daily dose of AMD3100 $(5 \mathrm{mg} / \mathrm{kg})$ starting the day after tumor cell injection. After 14 days, mice were sacrificed and the livers were collected for tumor burden analysis by quantifying the liver tissue area occupied by metastatic foci. Daily treatment with AMD3100 antagonist resulted in a significant reduction in the tumor burden in the liver of tumor-bearing mice compared to those treated with vehicle solution (Fig. 4A). In fact, AMD3100-treated tumor-bearing mice showed a 2-fold decrease in the metastatic area occupied in the liver along with a reduced number of foci of every size, and a significant reduction of small foci counts (Fig. 4B and C). Therefore, blocking CXCR4 is linked with reduced liver metastatic growth of colorectal C26 cancer cells in mice.

aSMA-expressing cell recruitment is decreased within liver foci in the AMD3100-treated mice. Activated HSCs express aSMA and help support angiogenesis (10). To confirm the obtained in vitro results, we analyzed the infiltration of $\alpha$ SMA-expressing cells in the tumor foci of the vehicle and AMD3100-treated mice 14 days after tumor cell injection. The immunohistochemical analyses revealed a significant 2 -fold decrease in the area positively stained for $\alpha$ SMA within the tumor foci in the liver of the AMD3100-treated mice compared to that of the vehicle-treated mice (Fig. 5A), which corroborates the in vitro results showing reduced migration

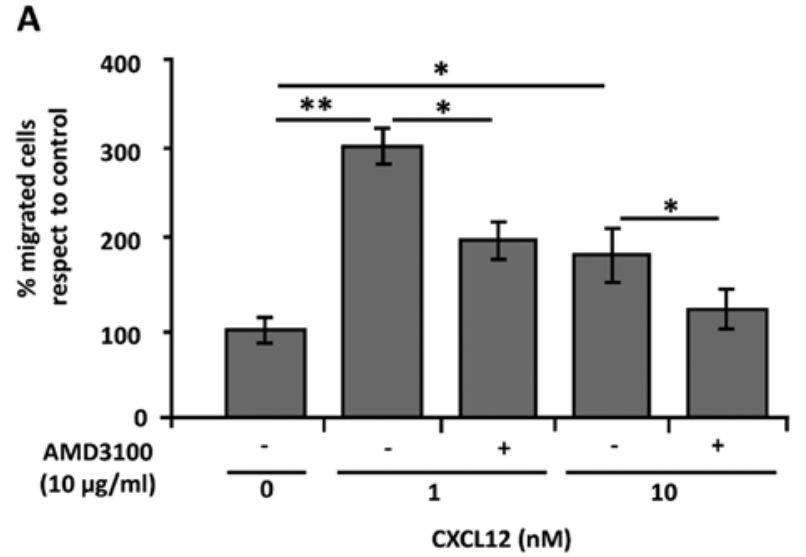

B

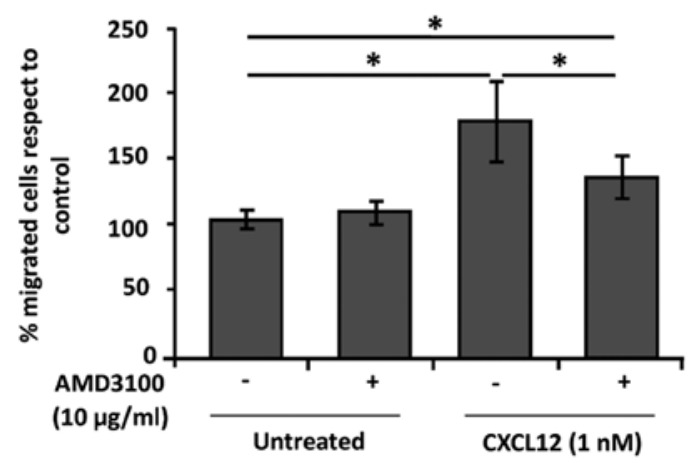

Figure 3. Effect of CXCL12 and AMD3100 on the migratory potential of C26 cancer cells and HSCs. (A) The migratory ability of C26 tumor cells was quantified after CXCL12 treatment at $24 \mathrm{~h}$. (B) Migration of control and CXCL12-treated HSCs. Differences were considered significant at ${ }^{*} \mathrm{P}<0.05$ and ${ }^{* *} \mathrm{P}<0.05$ 
A
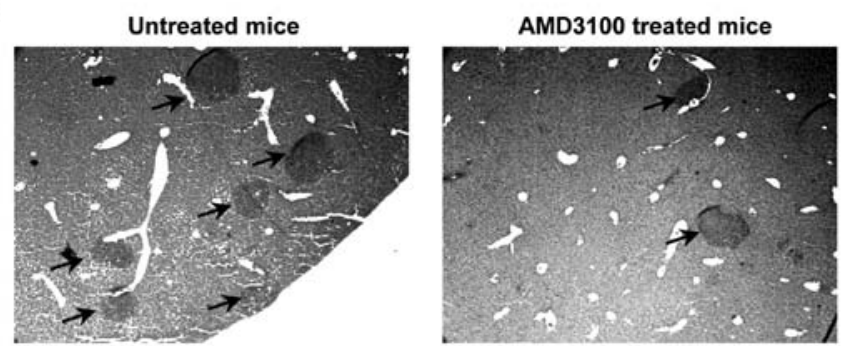

B

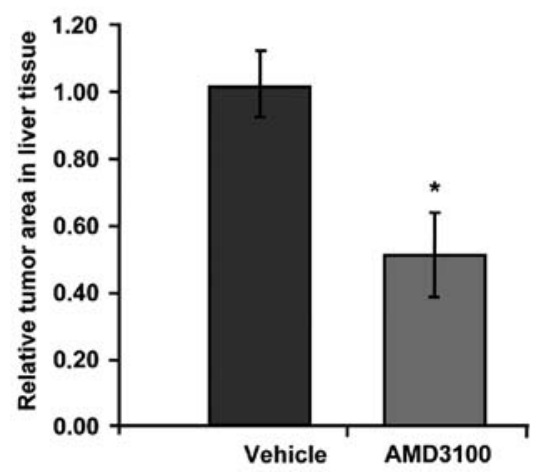

C

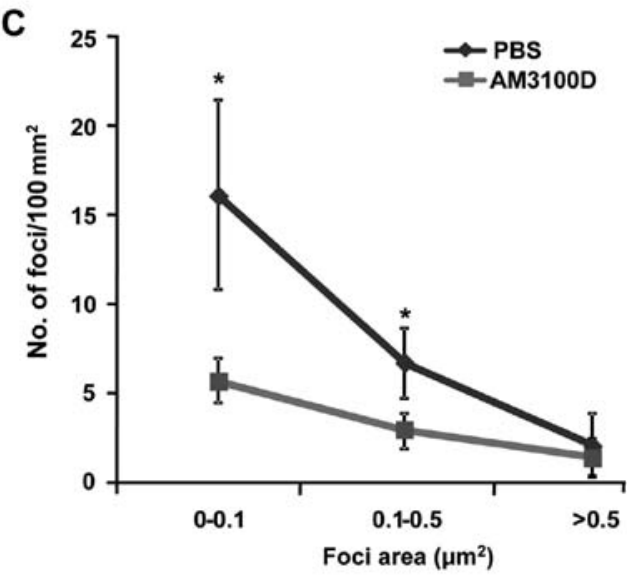

Figure 4. Metastatic area of tumor-bearing vehicle- and AMD3100-treated mice. Mice injected with $2 \times 10^{5}$ tumor cells were sacrificed 14 days after tumor cell injection and livers were collected for histological analysis. (A) Histological images of liver tissue from untreated and AMD3100 (5 mg/kg)-treated tumor-bearing mice. (B) The liver area occupied by tumor cells was quantified and is represented as the percentage of liver area occupied by the tumor foci. (C) Quantification of the number of foci in relation to area. Differences were considered statistically significant at ${ }^{*} \mathrm{P}<0.05$.
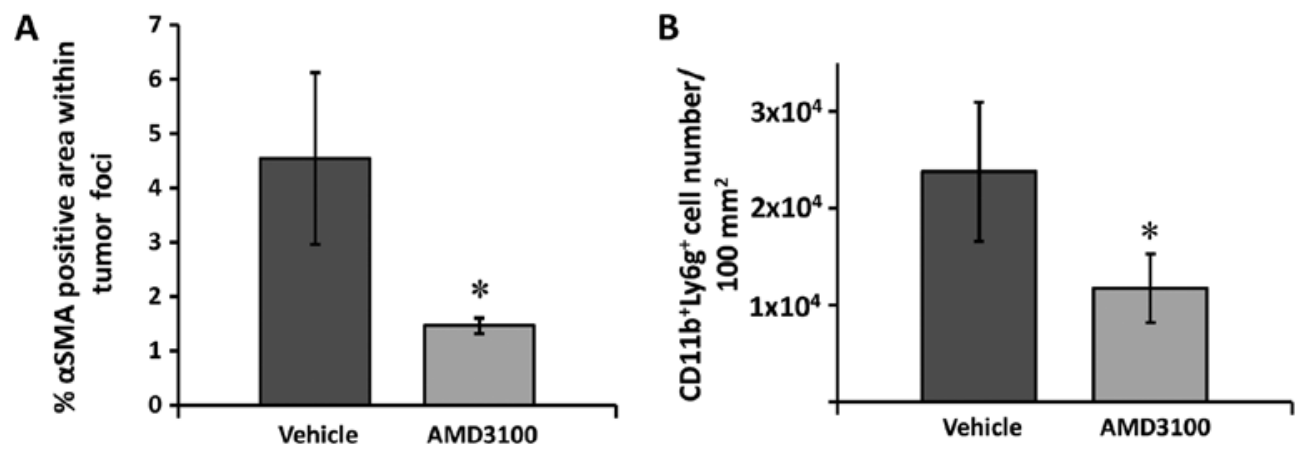

Figure 5. Effect of AMD3100 on $\alpha \mathrm{SMA}$-expressing cell and $\mathrm{CD} 11^{+} \mathrm{Ly}_{6 \mathrm{G}}{ }^{+}$cell recruitment into tumor foci. Mice were sacrificed 14 days after tumor cell injection and livers were collected for immunohistological analysis. (A) The area occupied by tumor-infiltrating $\alpha$ SMA-expressing cells was quantified. Cell nuclei were stained using DAPI. (B) The number of double-positive CD11b ${ }^{+}$Ly $6 \mathrm{G}^{+}$cells was assessed in the peritumoral areas. The mean of 10 different $\mathrm{x} 10$ fields is represented (10 livers/group). Differences were considered statistically significant at ${ }^{*} \mathrm{P}<0.05$.

of HSCs uponantagonist treatment. Hence, HSC expression of CXCR4 appears to be involved in chemotaxis leading to the recruitment of HSCs into tumor areas.

AMD3100 treatment is related to reduced $C D 11 b^{+} L y 6 G^{+}$ MDSC recruitment into the metastatic liver. HSCs are known to promote immune suppression via different pathways (11).In fact, evidence supports their role in the induction of MDSCs (21), which may drive immune suppression in the tumor microenvironment. These immune cells express CXCR4, and their chemotactic migration towards CXCL12 has been reported. Therefore, we investigated the effect of AMD3100 on the recruitment of $\mathrm{CD}_{11 \mathrm{~b}} \mathrm{Ly}_{6 \mathrm{G}}{ }^{+} \mathrm{MDSC}$ into the metastatic liver. The quantification of CD11b $\mathrm{Ly}^{+} \mathrm{G}^{+} \mathrm{MDSC}$ in tumor-bearing mice revealed a marked 2 -fold decrease in the number of positive cells in the liver of the AMD3100-treated mice, compared to that of the vehicle-treated mice (Fig. 5B), which is in line with the decrease in $\alpha$ SMA-positive cells in the tumor foci in the AMD3100-treated tumor-bearing mice. This observation lets us conclude that the infiltration of MDSCs into the metastatic liver may be mediated by expression of CXCR4.

Circulating $C D 11 b^{+} L y 6 G^{+}$number is reduced in the AMD3100-treated mice. The increase in MDSCs in the liver could represent an induction in the target organ but could also reflect an increase in the circulating number of MDSCs. Giving the influence of HSC-derived factors in these cells, the differentiation of the circulating pool could be compromised 


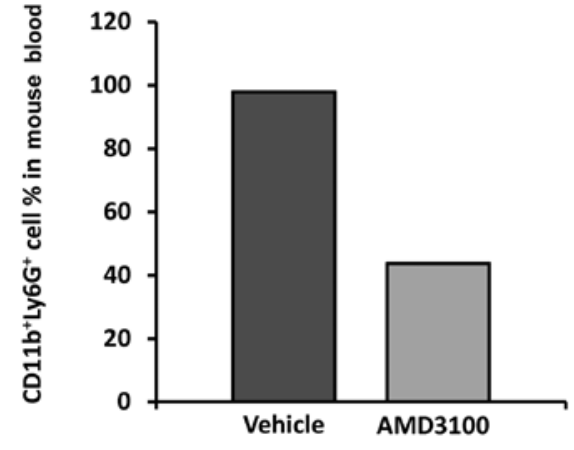

Figure 6. Effect of AMD3100 treatment on the count of CD11b+Ly6G ${ }^{+}$cells in the blood of tumor-bearing mice. Flow cytometric analyses from mouse blood revealed differences in the percentage of double-positive cells in the blood of untreated and AMD3100-treated mice. Double-positive cells for $\mathrm{CD} 11 \mathrm{~b}^{+} \mathrm{Ly}_{6 \mathrm{G}} \mathrm{G}^{+}$in tumor-bearing untreated mice are represented in the dark grey column while AMD3100-treated mouse double-positive cells are represented in the light grey column.

due to attenuated HSC activation and infiltration into the metastatic lesions. In order to assess the rate of blood born MDSCs, tumor-bearing mice treated with AMD3100 or vehicle solution were sacrificed after 14 days of tumor cell inoculation and blood was collected from 4 animals per experimental group for PMNC isolation. Then, the presence of double-positive CD $11 b^{+}{\text {Ly } 6 G^{+}}$cell percentage was calculated by flow cytometry. The results revealed a significant 2 -fold decrease

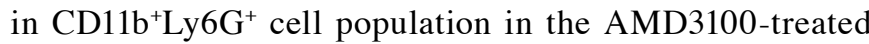
tumor-bearing mice, compared to the mice treated with vehicle where almost $98 \%$ of $\mathrm{Ly}_{6 \mathrm{G}}{ }^{+}$cells were positive for the monocytic marker CD11b. In contrast, only $43.8 \%$ of Ly $6 \mathrm{G}^{+}$cells exhibited a double-positive phenotype in the AMD3100-treated tumo- bearing mice (Fig. 6). Consequently, blockage of CXCR4 and the subsequent response related to CXCL12 ligation promoted a reduced MDSC percentage in the blood of the AMD3100-treated mice.

\section{Discussion}

CXCR4 has been reported in several human and mouse cancer cell lines $(9,22,23)$. However, modulation of the expression of this receptor in the tumor microenvironment remains poorly characterized. CAFs have been shown to promote the expression of a wide variety of cell receptors and adhesion molecules.

Herein, we showed that soluble factors derived from control or tumor-activated HSCs increased the expression of CXCR4 in tumor cells. This increase may be mediated by the production of IL-1 and IL- 6 by HSCs, which has been shown to promote CXCR4 upregulation in different cell types $(24,25)$. Moreover, both human and mouse HSCs express CXCR4. In fact, the increased expression of this receptor has been linked to progressive HSC activation under culture conditions. Furthermore, the upregulation of CXCR4 has also been reported in fibrotic livers, primarily related to activated HSCs (26). Interestingly, HSC activation has also been observed during metastatic colonization of the liver (10). Consistent with these findings, we reported upregulated CXCR4 expression in tumor-activated HSCs in vitro.

The metastatic process is orchestrated by circulating tumor cells in concert with cells of the target organ. Tumor cells migrate towards soluble stimulus, which attract them to the colonizing organ. Among others, CXCL12, the main lig and for CXCR4, has been reported to drive tumor cell migration and has been shown to be upregulated in the liver under pathological conditions, including cancer (27). In line with several reports, our results showed that $\mathrm{C} 26$ migration was increased after CXCL12 stimulation (25) whereas C26 treatment with CXCR4 antagonist AMD3100 led to attenuated response to this chemokine. This phenomenon could be in part mediated by Rho and Rac GTPases, involved in metastatic colonization, as observed in colon cancer and hepatoma cells after CXCR4-mediated CXCL12 stimulation (28), therefore, promoting cancer cell extravasation. Indeed, the formation of pseudopodia was described in CXCL12-treated multiple myeloma cells (29), which may also account for the facilitation of tumor migration by CXCL12-treated C26 cells.

Once in a target organ, cancer cells begin to proliferate in an uncontrolled manner driving tumor growth. Target organ cells support the tumor growth by the secretion of a wide variety of growth factors, cytokines and chemokines. In the liver, CXCL12 is produced by HSCs and LSECs in addition to malignant cells (30). Activated LSECs and HSCs secrete CXCL12, which may have an effect on CXCR4-expressing metastasizing colorectal cancer cells. Our results showed, however, that CXCL12 slightly increased the proliferation of C26 cells, a response that could be more pronounced in vivo, since stromal cells stimulate CXCR4 expression in C26 cells. The activation of the MAPK pathway could be responsible for this proliferation enhancement, as previously reported in other cell types (31). Moreover, this chemokine switches on the PI3K/AKT pathway, driving tumor growth (32), which could account for the increased $\mathrm{C} 26$ cancer cell viability.

Tumor growth is usually accompanied by ECM remodeling, a required step for disease progression. ECM remodeling is carried out by both tumor and stromal cells, such as HSCs in the liver. Intriguingly, the migration of HSCs was also fostered after treatment with this chemokine, which was partially abrogated after blocking CXCR4 using AMD3100. After interaction of $\mathrm{C} 26$ cells with liver sinusoidal endothelial cells (LSECs), Valcarcel et al observed an increase in VEGF, known to be an upstream inductor of CXCL12 in the liver (33), along with the initiation of an inflammatory response (20) that may account for HSC recruitment in tumor foci. This observation agrees with previous reports linking stimulated migration ability of human primary HSCs treated with CXCL12, along with CXCL12-mediated contraction mechanism in mouse primary HSC line, which may play a significant role in this process $(34,35)$. Surprisingly, we observed no changes in MMP-2 or MMP-9 activation in both C26 and HSCs treated with CXCL12, in line with other groups reporting no effect in CXCL12-treated cells (36). This result could be explained by the fact that HSCs do secrete CXCL12, which could activate in an autocrine manner the secretion of MMPs (37). In concordance with these results, we also observed a decrease in the expression of MMP-2 in these cells after AMD31000 treatment, which avoids the interaction of CXCL12 with its receptor CXCR4.

The production of CXCL12 in the liver is elevated (38) and this organ becomes a perfect collecting site for CXCR4-expressing circulating cells. Thus, this might be 
one of the reasons why the liver represents one of the main target organs for metastatic colorectal cancer cells. In fact, the administration of CXCR4 antagonist AMD3100 in colorectal liver metastasis model reduced by 2 -fold the metastatic area in the liver. Several reports have shown similar results by hijacking the circulating CXCL12 or administering AMD3100 into nude mice, therefore, impairing the metastatic colonization of the liver by tumor cells. The CXCR4-CXCL12 interaction mediated proliferative response observed in colorectal cancer cells $(39,40)$ can be blocked by AMD3100 administration in the tumor-microenvironment, contributing to the reported decrease in the metastatic area of AMD3100-treated tumor-bearing mice. This observation may be further mediated by reduced aSMA-expressing cells into metastatic foci, which impairs the angiogenic response and, consequently, the blood-mediated oxygen and element supply to the tumor mass, a required step for disease development. In addition, CXCR4-CXCL12 interplay has been shown to be responsible for elevated VEGF availability in tumor areas, driving new vessel formation (41). Interestingly, this group reported that HSCs mediated VEGF upregulation during liver metastasis, which may facilitate LSEC recruitment and transition from avascular to vascular stage of metastatic foci (10). Therefore, AMD3100 administration might interfere in the VEGF secretion by CXCL12-activated HSCs, leading to impaired neovascularization due to deficient LSEC recruitment.

These activated myofibroblast-like cells secrete a wide array of cytokines, such as, IL-1, IL-6 or IL-10 (42). IL-1 cytokine, along with VEGF, promote the differentiation and generation of MDSCs from the bone marrow, a highly relevant immunosuppressive cell population, profoundly linked with cancer progression and immune tolerance. Chou et al showed not only that HSCs can promote generation of MDSCs but also that these MDSCs possess a potent immune inhibitory activity (43). Our results showed that the proportion of circulating $\mathrm{CD} 11 \mathrm{~b}^{+} \mathrm{Ly} 6 \mathrm{G}^{+}$cells was elevated in untreated tumor-bearing mice, compared to those mice treated with AMD3100. This decrease in MDSCs may be due to the down regulation of differentiation stimuli secreted by both tumor cells and HSCs when blocking CXCR4, hence, leading to lower numbers of MDSCs. In fact, these CD11b $\mathrm{Ly}^{+} \mathrm{G}^{+}$cells express CXCR4 and migrate towards CXCL12 (44), therefore, accumulating in tumor areas. Our in vivo studies revealed that blocking CXCR4 drove to an attenuated recruitment of this suppressive cell subset into metastatic liver, in concordance with several studies reporting decreased numbers of these cells in breast carcinoma or subcutaneous lesions of hepatoma and breast carcinoma cells using CXCR4 antagonists $(45,46)$. This is also in close relation with the reduced number of HSCs recruited to the tumor foci after blockage of CXCR4 signaling by AMD3100. Interestingly, the soluble factors produced after C26 interaction with LSECs recruit HSCs through a COX-2 mediated pathway (data not shown), and the same pathway is required for MDSC accumulation driven by recruited activated HSCs (21).

Taken together, our data uncovered the relevance of tumor and stromal crosstalk driving CXCR4-CXCL12 interplay stimulation, which in turn favors the recruitment of tumor cells and MDSCs into the liver. This is in relation to the infiltration of $\alpha$ SMA-expressing activated HSCs into metastatic foci which fosters the recruitment and accumulation of differentiated CD11b $\mathrm{Ly}^{+} \mathrm{g}^{+}$cell subsets from the circulation of tumor-bearing mice, driving liver metastasis. All of these processes are reduced by the administration of AMD3100, implying CXCR4/CXCL12 interaction as a perfect target for the inactivation of multiple pro-tumoral processes involving both tumor and host cells.

\section{Acknowledgements}

This study was financially supported in part by a postdoctoral fellowship from the University of the Basque Country to A.B. and by funds from the Basque Government-Saiotek to B.A.

\section{Competing interests}

The authors declare that they have no competing interests.

\section{References}

1. Van den Eynden GG, Majeed AW, Illemann M, Vermeulen PB Bird NC, Høyer-Hansen G, Eefsen RL, Reynolds AR and Brodt P: The multifaceted role of the microenvironment in liver metastasis: Biology and clinical implications. Cancer Res 73: 2031-2043, 2013.

2. Colangelo T, Polcaro G, Muccillo L, D'Agostino G, Rosato V, Ziccardi P, Lupo A, Mazzoccoli G, Sabatino L and Colantuoni V: Friend or foe? The tumour microenvironment dilemma in colorectal cancer. Biochim Biophys Acta 1867: 1-18, 2017.

3. Trautmann F, Cojoc M, Kurth I, Melin N, Bouchez LC, Dubrovska A and Peitzsch C: CXCR4 as biomarker for radioresistant cancer stem cells. Int J Radiat Biol 90: 687-699, 2014.

4. Brand S, Dambacher J, Beigel F, Olszak T, Diebold J, Otte JM, Göke B and Eichhorst ST: CXCR4 and CXCL12 are inversely expressed in colorectal cancer cells and modulate cancer cell migration, invasion and MMP-9 activation. Exp Cell Res 310: $117-130,2005$.

5. Ottaiano A,Franco R,Aiello Talamanca A,Liguori G, TatangeloF, Delrio P, Nasti G, Barletta E, Facchini G, Daniele B, et al: Overexpression of both CXC chemokine receptor 4 and vascular endothelial growth factor proteins predicts early distant relapse in stage II-III colorectal cancer patients. Clin Cancer Res 12: 2795-2803, 2006.

6. Yamada S, Shimada M, Utsunomiya T, Morine Y, Imura S, Ikemoto T, Mori H, Arakawa Y, Kanamoto M, Iwahashi S and Saito Y: CXC receptor 4 and stromal cell-derived factor 1 in primary tumors and liver metastases of colorectal cancer. J Surg Res 187: 107-112, 2014.

7. Li JK, Yu L, Shen Y, Zhou LS, Wang YC and Zhang JH: Inhibition of CXCR4 activity with AMD3100 decreases invasion of human colorectal cancer cells in vitro. World J Gastroenterol 14: 2308-2313, 2008

8. Wendel C, Hemping-Bovenkerk A, Krasnyanska J, Mees ST, Kochetkova M, Stoeppeler S and Haier J: CXCR4/CXCL12 participate in extravasation of metastasizing breast cancer cells within the liver in a rat model. PLoS One 7: e30046, 2012.

9. O'Boyle G, Swidenbank I, Marshall H, Barker CE, Armstrong J, White SA, Fricker SP, Plummer R, Wright M and Lovat PE: Inhibition of CXCR4-CXCL12 chemotax is in melanoma by AMD11070. Br J Cancer 108: 1634-1640, 2013.

10. Olaso E, Salado C, Egilegor E, Gutierrez V, Santisteban A, Sancho-Bru P,Friedman SL and Vidal-Vanaclocha F: Proangiogenic role of tumor-activated hepatic stellate cells in experimental melanoma metastasis. Hepatology 37: 674-685, 2003.

11. Puche JE, Saiman Y and Friedman SL: Hepatic stellate cells and liver fibrosis. Compr Physiol 3: 1473-1492, 2013.

12. Song Y, Kim SH, Kim KM, Choi EK, Kim J and Seo HR: Activated hepatic stellate cells play pivotal roles in hepatocellular carcinoma cell chemoresistance and migration in multicellular tumor spheroids. Sci Rep 6: 36750, 2016.

13. Cojoc M, Peitzsch C, Trautmann F, Polishchuk L, Telegeev GD and Dubrovska A: Emerging targets in cancer management: role of the CXCL12/CXCR4 axis. Onco Targets Ther 6: 1347-1361, 2013. 
14. Mishra P, Banerjee D and Ben-Baruch A: Chemokines at the crossroads of tumor-fibroblast interactions that promote malignancy. J Leukoc Biol 89: 31-39, 2011.

15. Kusmartsev S and Gabrilovich DI: Effect of tumor-derived cytokines and growth factors on differentiation and immune suppressive features of myeloid cells in cancer. Cancer Metastasis Rev 25: 323-331, 2006.

16. Zhang H, Li Z, Wang L, Tian G, Tian J, Yang Z, Cao G, Zhou H, Zhao L, Wu Z and Yin Z: Critical role of myeloid-derived suppressor cells in tumor-induced liver immune suppression through inhibition of NKT cell function. Front Immunol 8: 129, 2017.

17. Goodwin TJ, Zhou Y, Musetti SN, Liu R and Huang L: Local and transient gene expression primes the liver to resist cancer metastasis. Sci Transl Med 8: 364ra153, 2016.

18. D'Alterio C, Barbieri A, Portella L, Palma G, Polimeno M, Riccio A, Ieranò C, Franco R, Scognamiglio G, Bryce J, et al: Inhibition of stromal CXCR4 impairs development of lung metastases. Cancer Immunol Immunother 61: 1713-1720, 2012.

19. Smedsrød B and Pertoft H: Preparation of pure hepatocytes and reticuloendothelial cells in high yield from a single rat liver by means of Percoll centrifugation and selective adherence. J Leukoc Biol 38: 213-230, 1985.

20. Arteta B, Lasuen N, Lopategi A, Sveinbjörnsson B, Smedsrød B and Vidal-Vanaclocha F: Colon carcinoma cell interaction with liver sinusoidal endothelium inhibits organ-specific antitumor immunity through interleukin-1-induced mannose receptor in mice. Hepatology 51: 2172-2182, 2010.

21. Xu Y, Zhao W, Xu J, Li J, Hong Z, Yin Z and Wang X: Activated hepatic stellate cells promote liver cancer by induction of myeloid-derived suppressor cells through cyclooxygenase- 2 . Oncotarget 7: 8866-8878, 2016.

22. Zhang SS, Han ZP, Jing YY, Tao SF, Li TJ, Wang H, Wang Y, Li R, Yang Y, Zhao X, et al: CD133(+)CXCR4(+) colon cancer cells exhibit metastatic potential and predict poor prognosis of patients. BMC Med 10: 85, 2012.

23. Kollmar O, Rupertus K, Scheuer C, Junker B, Tilton B, Schilling MK and Menger MD: Stromal cell-derived factor-1 promotes cell migration and tumor growth of colorectal metastasis. Neoplasia 9: 862-870, 2007.

24. Oh JW, Drabik K, Kutsch O, Choi C, Tousson A and Benveniste EN: CXC chemokine receptor 4 expression and function in human astroglioma cells. J Immunol 166: 2695-2704, 2001.

25. Odemis V, Moepps B, Gierschik P and Engele J: Interleukin- 6 and cAMP induce stromal cell-derived factor-1 chemotaxis in astroglia by up-regulating CXCR4 cell surface expression. Implications for brain inflammation. J Biol Chem 277: 39801-39808, 2002.

26. Hong F, Tuyama A, Lee TF, Loke J, Agarwal R, Cheng X, Garg A, Fiel MI, Schwartz M, Walewski J, et al: Hepatic stellate cells express functional CXCR4: Role in stromal cell-derived factor-1alpha-mediated stellate cell activation. Hepatology 49: 2055-2067, 2009.

27. Sun X, Cheng G, Hao M, Zheng J, Zhou X,Zhang J, Taichman RS, Pienta KJ and Wang J: CXCL12/CXCR4/CXCR7 chemokine axis and cancer progression. Cancer Metastasis Rev 29: 709-722, 2010.

28. Gassmann P, Haier J, Schlüter K, Domikowsky B, Wendel C Wiesner U, Kubitza R, Engers R, Schneider SW, Homey B and Müller A: CXCR4 regulates the early extravasation of metastatic tumor cells in vivo. Neoplasia 11: 651-661, 2009.

29. Alsayed Y, Ngo H, Runnels J, Leleu X, Singha UK, Pitsillides CM, Spencer JA, Kimlinger T, Ghobrial JM, Jia X, et al: Mechanisms of regulation of CXCR4/SDF-1 (CXCL12)-dependent migration and homing in multiple myeloma. Blood 109: 2708-2717, 2007.

30. Marra F and Tacke F: Roles for chemokines in liver disease. Gastroenterology 147: 577-594.e1, 2014.
31. Wu Y, Peng H, Cui M, Whitney NP, Huang Y and Zheng JC: CXCL12 increases human neural progenitor cell proliferation through Akt-1/FOXO3a signaling pathway. J Neurochem 109: 1157-1167, 2009.

32. Heinrich EL, Lee W, Lu J, Lowy AM and Kim J: Chemokine CXCL12 activates dual CXCR4 and CXCR7-mediated signaling pathways in pancreatic cancer cells. J Transl Med 10: 68, 2012.

33. Valcárcel M, Arteta B, Jaureguibeitia A, Lopategi A, Martínez I, Mendoza L, Muruzabal FJ, Salado C and Vidal-Vanaclocha F: Three-dimensional growth as multicellular spheroid activates the proangiogenic phenotype of colorectal carcinoma cells via LFA-1-dependent VEGF: Implications on hepatic micrometastasis. J Transl Med 6: 57, 2008.

34. Saiman Y, Agarwal R, Hickman DA, Fausther M, El-Shamy A, Dranoff JA, Friedman SL and Bansal MB: CXCL12 induces hepatic stellate cell contraction through a calcium-independent pathway. Am J Physiol Gastrointest Liver Physiol 305: G375-G382, 2013.

35. Yuan X, Xiao T, Dai F, Yu Y and Lou J: CXCL12 $\alpha$ and CXCL12 $\beta$ stimulate the migration of cultured LX-2 hepatic stellate cells. Xi Bao Yu Fen Zi Mian Yi Xue Za Zhi 31: 293-296, 2015 (In Chinese).

36. Zhang J, Sarkar S and Yong VW: The chemokine stromal cell derived factor-1 (CXCL12) promotes glioma invasivenessthrough MT2-matrix metalloproteinase. Carcinogenesis 26: 2069-2077, 2005.

37. Teng F, Tian WY, Wang YM, Zhang YF, Guo F, Zhao J, Gao C and Xue FX: Cancer-associated fibroblasts promote the progression of endometrial cancer via the SDF-1/CXCR4 axis. J Hematol Oncol 9: 8, 2016.

38. Sun X, Cheng G, Hao M, Zheng J, Zhou X, Zhang J, Taichman RS, Pienta KJ and Wang J: CXCL12/CXCR4/CXCR7 chemokine axis and cancer progression. Cancer Metastasis Rev 29: 709-722, 2010.

39. Ma L, Qiao H, He C, Yang Q, Cheung CH, Kanwar JR and Sun X: Modulating the interaction of CXCR4 and CXCL12 by low-molecular-weight heparin inhibits hepatic metastasis of colon cancer. Invest New Drugs 30: 508-517, 2012.

40. Heckmann D, Maier P, Laufs S, Li L, Sleeman JP, Trunk MJ, Leupold JH, Wenz F, Zeller WJ, Fruehauf S and Allgayer H: The disparate twins: A comparative study of CXCR4 and CXCR7 in SDF-1 $\alpha$-induced gene expression, invasion and chemosensitivity of colon cancer. Clin Cancer Res 20: 604-616, 2014.

41. Liang Z, Brooks J, Willard M, Liang K, Yoon Y, Kang S and Shim H: CXCR4/CXCL12 axis promotes VEGF-mediated tumor angiogenesis through Akt signaling pathway. Biochem Biophys Res Commun 359: 716-722, 2007.

42. Lang A, Sakhnini E, Fidder HH, Maor Y, Bar-Meir S and Chowers Y: Somatostatin inhibits pro-inflammatory cytokine secretion from rat hepatic stellate cells. Liver Int 25: 808-816, 2005.

43. Chou HS, Hsieh CC, Yang HR, Wang L, Arakawa Y, Brown K, Wu Q, Lin F, Peters M, Fung JJ, et al: Hepatic stellate cells regulate immune response by way of induction of myeloid suppressor cells in mice. Hepatology 53: 1007-1019, 2011.

44. Sawanobori Y, Ueha S, Kurachi M, Shimaoka T, Talmadge JE Abe J, Shono Y, Kitabatake M, Kakimi K, Mukaida N and Matsushima K: Chemokine-mediated rapid turnover of myeloid-derived suppressor cells in tumor-bearing mice. Blood 111: 5457-5466, 2008.

45. Yang L, Huang J, Ren X, Gorska AE, Chytil A, Aakre M, Carbone DP, Matrisian LM, Richmond A, Lin PC and Moses HL: Abrogation of TGF beta signaling in mammary carcinomas recruits $\mathrm{Gr}-1^{+} \mathrm{CD} 11 \mathrm{~b}^{+}$myeloid cells that promote metastasis. Cancer Cell 13: 23-35, 2008.

46. Ba H, Li B, Li X, Li C, Feng A, Zhu Y, Wang J, Li Z and Yin B: Transmembrane tumor necrosis factor- $\alpha$ promotes the recruitment of MDSCs to tumor tissue by upregulating CXCR4 expression via TNFR2. Int Immunopharmacol 44: 143-152, 2017. 